Objective To determine relationship between occupational exposure to benzene-toluene-xylene mixture (BTX) and IL-10, TNF and IL-12 production by peripheral blood mononuclear cells.

Methods Exposure was estimated in 54 workers from a paint company in Mexico City through BTX accumulated potential dose (BTX-APD). Two exposure groups were formed: high and low BTX-APD established with a cutoff point at $\geq 1.0$ of BTX$\mathrm{APD}$, as a function of the geometric mean of the estimator's value distribution and the higher agreement between BTX-APD $\geq 1.0$ and the areas referred as using (or not) organic solvents in the work process. IL-10, TNF and IL-12 concentrations were measured with ELISA. Through multiple linear regression models, the production of each of the proposed cytokines and of the whole set was assessed.

Results Workers with high BTX-APD showed a significant reduction in TNF production $(\beta=-1,196.0 \mathrm{pg} / \mathrm{mL} ; \mathrm{p}=0.01)$; a reduction for IL-10 $(\beta=-520.3 ; \mathrm{p}=0.13)$ and IL-12 $(\beta=-843.3 ; p=0.09)$ was also observed, although without statistical significance.

Conclusions TNF production assessed in workers with a high BTX-APD is lower than in those with a low BTX-APD, but not in IL-10 and IL-12 production.

\section{BENCHMARK DOSE ESTIMATION OF HEMATOTOXICITY AND GENOTOXICITY AMONG CHINESE BENZENE EXPOSED WORKERS IN SHOE FACTORIES}

GH Zhang. School of Public Health, Fudan University, Shanghai 200032, China

10.1136/oemed-2013-101717.112

Objectives Benzene exposure can induce hematotoxicity and hematotoxicity at occupational exposure level below $1 \mathrm{ppm}$ according to previous reports. The purpose of this study was to calculate benchmark dose (BMD) for chromosomal damage and reduced white blood cell (WBC) induced by benzene among the exposed workers in Wenzhou, China.

Methods A group of 317 workers occupationally exposed to benzene and 102 unexposed workers were examined for hematotoxicity indicated by WBC count, and for genotoxicity measured by cytokinesis- blocked micronucleus (CBMN) assay with peripheral blood lymphocytes. The cumulative exposure dose (CED) of benzene was calculated basing on the job type and duration of each job and the benzene concentration in workplace. Benchmark Dose Software (BMDS) Version2.2.1 (US EPA) was used to calculate the BMD and its lower confidence limit, BMDL.

Results demonstrated that there was a strong dose-response relationship between benzene CED and the effect biomarkers (the MN frequency and WBC count). The BMDL10 by CBMN frequency were found to be 5.16, 1.84 and 2.35 ppm-year for benzene -exposed male, benzene -exposed female and total exposed workers, and 5.45, 3.94, 10.25 ppm-year by WBC count, respectively.

Conclusions $2 \mathrm{ppm}$ for chromosomal damage (CBMN) and 4 ppm for hematotoxicity (WBC) of occupational exposure limits of benzene were suggested according to our findings. Further studies need to be confirmed and validated.

\section{FERRITIN MAY PREDICT 5-YEAR RISK OF METABOLIC SYNDROME IN TAIWANESE NON-OBESE MALE WORKERS: INSIGHT FROM AN OCCUPATIONAL COHORT STUDY}

1J H Lee, 'Chang, ${ }^{2}$ Wang, 'Liou, ${ }^{3}$ Hsiao. 'National Health Research Institutes, Zhuna, Taiwan; ${ }^{2}$ Department of Public Health, College of Medicine, National Cheng Kung University, Tainan, Taiwan; ${ }^{3}$ Hsiao's Charity Clinic, Taoyuan, Taiwan

\subsection{6/oemed-2013-101717.113}

Objectives To investigate association between ferritin and 5-year risk of developing metabolic syndrome (MetS) among apparently healthy middle-aged male workers.

Methods We established a prosepective cohort study in an electronic-manufacturing factory by using a detailed medical checkup program in 2002, and followed up them with a health examination in 2007. Each individual underwent physical examination and blood biochemistry tests; body mass index (BMI), blood pressure, and waist circumference were measured by the registered nurses. We collected data from self-reported questionnaires, ferritin, insulin resistance estimated by homeostasis model assessment (HOMA), and fatty liver revealed by abdominal ultrasound in 2002, and aimed to explore their association with risk of metabolic abnormalities in 2007. MetS was diagnosed according to the modified National Cholesterol Education Program Adult Treatment Panel III criteria accounting for Asia Pacific/Taiwanese population. Cox proportional hazard models were applied to discover if ferritin is a predictor for development of MetS.

Results A total of 1493 workers were recruited in this study. Most subjects were males (73.3\%) with a baseline mean (SD) age of $32.5(6.0)$. Baseline MetS was diagnosed in $21.5 \%$ of males, and $14.8 \%$ of females. The prevalence of MetS after 5 years significantly increased with the tertiles of baseline ferritin for both genders. In males, ferritin $>200 \mathrm{mcg} / \mathrm{L}$ was associated with increased risk of MetS. Within 5-year follow-up, incident 114 cases of MetS developed among 877 MetS-free males. Among the non-obese males $(\mathrm{BMI}<25)$, ferritin $>200 \mathrm{mcg} / \mathrm{L}$ may predict MetS with a hazard ratio (HR) of $2.23(95 \%$ C. I. 1.024.89) compared to the first tertile $(<123 \mathrm{mcg} / \mathrm{L})$ after controlling for age in the Cox models; non-alcoholic fatty liver diseases (NAFLD) was significantly related to new-onset MetS with a HR of 4.83 while a positively increased trend of higher tertiles of ferritin associated with MetS was observed.

\section{CANCER-RELATED PROTEINS IN LUNG TISSUE FROM URANIUM MINERS - VARIATION BY OCCUPATIONAL EXPOSURE AND SUBTYPE OF LUNG CANCER}

'S C Casjens, ${ }^{2}$ Stricker, ${ }^{2}$ Westerwick, ${ }^{1}$ Taeger, ${ }^{1}$ Rabstein, ${ }^{1}$ Wiethege, ${ }^{2}$ Tannapfel, ${ }^{1}$ Brüning, 'Johnen, ${ }^{1}$ Pesch. 'Institute for Prevention and Occupational Medicine of the DGUV (IPA), Bochum, Germany; ${ }^{2}$ Institute of Pathology, Ruhr-Universität Bochum, Bochum, Germany

\subsection{6/oemed-2013-101717.114}

Objectives We investigated the association of cumulative exposure to radon and arsenic with cancer-specific proteins in lung tissue from uranium miners.

Methods Paraffin-embedded lung tissue of 147 miners was randomly selected from a biobank established for German uranium miners comprising adenocarcinoma (AdCa), squamous cell carcinoma (SqCC), small cell lung cancer (SCLC), and cancer-free tissue. Within each stratum, we additionally stratified by level of cumulative exposure to radon and arsenic. Lifetime exposure to radon and arsenic was estimated using a job-exposure matrix developed for uranium mining in Germany. For 22 cancerrelated proteins, immunohistochemical scores were calculated from the intensity and percentage of stained cells. The association of these scores with exposure to radon and arsenic was 
explored by Spearman rank correlation coefficients $\left(r_{s}\right)$. We further applied supervised and unsupervised classification to investigate protein patterns by type of lung tissue.

Results Occupational exposure was associated with an up-regulation of NOTCH1 (radon: $\mathrm{r}_{\mathrm{s}}=0.18,95 \%$ CI 0.02-0.33; arsenic: $\mathrm{r}_{\mathrm{s}}=0.23,95 \%$ CI $\left.0.07-0.38\right)$. MUC1 classified lung cancer from cancer-free tissue (failure rate of $2.1 \%$ ), and HIF1A and NKX2-1 discriminated the major subtypes of lung cancer with a failure rate of $8.4 \%$.

Conclusions These results suggest that the radiation-sensitive protein NOTCH1 can be up-regulated in lung tissue from uranium miners by level of exposure to pulmonary carcinogens. The distinct phenotypes of the major subtypes of lung cancer could be discriminated with cancer-related proteins.

\section{SALIVARY CORTISOL AND DEPRESSION - IS THERE AN ASSOCIATION IN A RANDOM SAMPLE OF PUBLIC SECTOR EMPLOYEES?}

${ }^{1} \mathrm{M}$ A V Agergaard Vammen, ${ }^{2}$ Thomsen, ${ }^{2}$ Mikkelsen, ${ }^{2}$ Bonde, ${ }^{3}$ Hansen, ${ }^{4}$ Andersen, ${ }^{5}$ Buttenschøn, ${ }^{4}$ Grynderup, ${ }^{4}$ Kolstad, ${ }^{6}$ Kærlev, ${ }^{4}$ Kærgaard, ${ }^{7}$ Rugulies, ${ }^{5}$ Mors. ${ }^{1}$ BisBispebjerg University Hospital, Copenhagen NV, Denmark; ${ }^{2}$ Department of Occupational and Environmental Medicine, Bispebjerg University Hospital, Copenhagen, Denmark; ${ }^{3}$ Department of Public Health, University of Copenhagen and National Research Centre, Copenhagen, Denmark; ${ }^{4}$ Danish Ramazzini Centre, Department of Occupational Medicine, Herning, Denmark; ${ }^{5}$ Centre for Psychiatric Research, Aarhus University Hospital, Risskov, Denmark; ${ }^{6}$ Center for National Clinical Databases South, Odense, Denmark; ${ }^{7}$ National Research Centre for the Working Environment, Copenhagen, Denmark

\subsection{6/oemed-2013-101717.115}

Objective To examine if salivary cortisol is associated with depressive symptoms and clinical depression among public sector employees.

Method In 2007, 10,036 public sector employees received a questionnaire along with salivary cortisol test tubes for home administration. A morning (30 min after awakening) and evening $(2000 \mathrm{~h})$ salivary sample were collected. 3,536 employees returned questionnaires and valid saliva samples. A subsample of participants $(n=387)$ collected three morning saliva samples (at awakening, 20min and 40min after awakening) plus an evening sample $(2000$ h). Participants were approached again in 2009 with questionnaire and salivary test tubes $(n=2,408)$. Participants with self-reported depressive symptoms (Common Mental Disorder Questionnaire) were invited to a SCAN interview (Schedules for Clinical Assessment in Neuropsychiatry, version 2.1) to determine clinical depression. The repeated cross-sectional data were analysed with logistic regression. Odds ratios of depressive symptoms and of clinical depression were estimated for morning, evening, mean and the difference between morning and evening cortisol (slope). For the subsample, CAR (awakening cortisol response) and AUC (the area under the curve) cortisol measures were calculated. We adjusted for gender, age, income, education, family history of depression, physical activity and alcohol consumption.

Results In 2007 the median level of cortisol was $12.5 \mathrm{nmol} / \mathrm{l}$ in the morning and $2.1 \mathrm{nmol} / \mathrm{l}$ in the evening. None of the measures of salivary cortisol were associated with self-reported depressive symptoms or clinical depression, neither in 2007 or 2009. E.g. in 2007, the odds ratios of depressive symptoms by a one unit increase in morning and evening cortisol $(\log [\mathrm{nmol} / \mathrm{litre}$ saliva]) were 0.97 (95\% CI: 0.83-1.13) and 1.05 (0.92-1.20), respectively, and of clinical depression 1.08 (95\% CI: 0.35-3.36) and $0.66(0.30-1.45)$, respectively.
Conclusion Salivary cortisol was not associated to self-reported symptoms of depression or to clinical depression.

\section{EFFECTS ON CORTISOL IN SALIVA FROM GOING TO WORK AND OTHER SALIVA SAMPLING CIRCUMSTANCES. RESULTS FROM A LARGE EPIDEMIOLOGICAL STUDY}

${ }^{1} \mathrm{~S}$ M Mikkelsen, ${ }^{1}$ Thomsen, ${ }^{2}$ Andersen, ${ }^{1}$ Bonde, ${ }^{3} \mathrm{Grynderup},{ }^{3} \mathrm{Kolstad},{ }^{2} \mathrm{~K}$ ærgaard, ${ }^{4} \mathrm{Kærlev}$, ${ }^{1}$ Vammen, ${ }^{5}$ Hansen. ${ }^{1}$ Bispebjerg University Hospital, Copenhagen, Denmark; ${ }^{2}$ Regional Hospital Herning, Herning, Denmark; ${ }^{3}$ Århus University Hospital, Århus, Denmark; ${ }^{4}$ Odense University Hospital, Odense, Denmark; ${ }^{5}$ National Research Centre for the Working Environment, Copenhagen, Denmark

\subsection{6/oemed-2013-101717.116}

Objectives To examine the effects of going to work and other saliva sampling circumstances on the concentration of cortisol in saliva.

Methods The study is a cross sectional population study of 3536 working persons with

data on cortisol concentrations in saliva samples, one in the morning and one in the evening, and information on saliva sampling circumstances recorded by questionnaire.

We studied the effects on cortisol from sampling on a work day compared to a day off; number of hours worked; smoking; leisure time physical activity; sleep problems the night before sampling and other sampling circumstances. These factors were included as covariates in ordinary least square regression analyses with the log of cortisol in saliva (nmol/l) as the dependent variable. We adjusted for effects of age, sex and saliva sampling times and time from awakening.

Results Saliva sampling times were the major determinants of cortisol concentrations in saliva, including linear and quadratic effects. Morning cortisol was 23\% higher on work days than on non-work days ( $\mathrm{p}<0.0001)$, controlling for sampling and awakening times and other potential confounders. This effect was independent of age, indicating that the acute and rather strong HPA axis response to an anticipated stressor (going to work) was not attenuated by almost daily repeats during many years of work. Working seven hours increased the mean of morning and evening cortisol by $11 \%(\mathrm{p}<0.0001)$. Smoking, leisure time physical activity and use of painkillers also had significant effects.

Conclusions The anticipation of going to work seems to elicit a rather strong acute increase in morning cortisol. This response was not attenuated by increasing age as one would expect if frequently repeated HPA-axis activations eventually leads to a reduced HPA-axis response to acute stressors.

\section{METABOLOMICS EXPERIMENT AMONG WORKERS EXPOSED TO 2, 3, 7, 8-TETRACHLORODIBENZO-P-DIOXIN (TCDD)}

${ }^{1} \mathrm{~F}$ Saberi Hosnijeh, ${ }^{2}$ Pechlivanis, ${ }^{2}$ Keun, ${ }^{3}$ Portengen, ${ }^{4}$ Bueno-de-Mesquita, ${ }^{3}$ Heederik, ${ }^{1}$ Vermeulen. ' Utrecht University, Utrecht, The Netherlands; ${ }^{2}$ Imperial College, London, United Kingdom; ${ }^{3}$ Institute for Risk Assessment Sciences (IRAS), Utrecht University, Utrecht, The Netherlands; ${ }^{4}$ National institute for public health and environment (RIVM), Bilthoven, The Netherlands

\subsection{6/oemed-2013-101717.117}

Objectives Previous occupational studies suggest that 2,3,7,8-tetrachlorodibenzo-p-dioxin (TCDD) exposure may be associated 